\title{
Reply to the Letter to the Editor: Quantitative evaluation of COVID-19 pneumonia severity by CT pneumonia analysis algorithm using deep learning technology and blood test results
}

\author{
Tomohisa Okuma ${ }^{1}$
}

Received: 12 July 2021 / Accepted: 14 July 2021 / Published online: 21 July 2021

C Japan Radiological Society 2021

We thank Drs. Mungmunpuntipantip and Wiwanitkit for their interest and valuable comments on our recent work [1]. As they pointed out, eGFR is calculated based on gender, age and serum creatinine [2], and we agree with them that the validity and reliability of eGFR estimation depends on accurate and precise measurements of serum creatinine. In this single-center study, serum creatinine was measured using a single system, which was based on the highly reliable and validated enzymatic method. The system was routinely calibrated during the study period to minimize intralaboratory variability.

For possible confounding factors on blood test results, we included all factors that could be retrospectively retrieved from our medical records. Although specimen collection and processing were not part of routine medical record keeping, many significant factors remained after Kruskal-Wallis univariate analysis as mentioned in Materials and methods. We thought it might be better to exclude independent variables with high correlation and examined for multicollinearity before conducting multivariate analysis. There was a correlation between age and eGFR $(r=-0.619)$ and between AST and LDH $(r=-0.693)$, and age and AST were excluded from the multivariate analysis. When we added age as a factor in multivariate analysis, eGFR and total HU mean were still independent factors. Thank you again for the important insights. Their suggestions are certainly worth considering in future multi-center prospective studies in this evolving field.

\section{Declarations}

Conflict of interest The authors declare that they have no conflict of interest.

\section{References}

1. Okuma T, Hamamoto S, Maebayashi T, Taniguchi A, Hirakawa K, Matsushita S, et al. Quantitative evaluation of COVID-19 pneumonia severity by CT pneumonia analysis algorithm using deep learning technology and blood test results. Jpn J Radiol. 2021. https://doi.org/10.1007/s11604-021-01134-4 ((Online ahead of print)).

2. Matsuo S, Imai E, Horio M, Yasuda Y, Tomita K, Nitta K, et al. Revised equations for estimated GFR from serum creatinine in Japan. Am J Kidney Dis. 2009;53:982-92.

Publisher's Note Springer Nature remains neutral with regard to jurisdictional claims in published maps and institutional affiliations.
Tomohisa Okuma

o-kuma@msic.med.osaka-cu.ac.jp

1 Department of Diagnostic Radiology, Osaka City General Hospital, 2-13-22 Miyakojima-hondori, Miyakojima-ku, Osaka 534-0021, Japan 